\title{
Bluetooth Düşük Enerji Teknolojisine Sahip İşaretçi ve Akıllı Telefon Temelli Öğrenci Yoklama Sistemi
}

\author{
Cüneyt BAYILMIŞ, Mehmet ÖZDEMİR \\ Sakarya Üniversitesi, Bilgisayar ve Bilişim Bilimleri Fakültesi, Bilgisayar Mühendisliği \\ cbayilmis@sakarya.edu.tr, ozdemirmehmt@gmail.com \\ (Geliş/Received: 04.04.2016; Kabul/Accepted: 09.06.2016) \\ DOI: $10.17671 /$ btd.94742
}

\begin{abstract}
Özet - Bluetooth düşük enerji teknolojisine sahip işaretçi (BLE beacon) cihazların kullanımı düşük enerji tüketimi, düşük maliyet ve hem iOS hem de Android işletim sistemi desteği nedeniyle hızla artmaktadır. Akıllı telefon, tablet gibi mobil cihazlar işaretçi cihazların konumlandırıldıkları alana geldiklerinde, işaretçi cihazlar tarafından gönderilen tanıtım mesajlarını alarak ilgili mobil uygulamalarını otomatik olarak çalıştırırlar. Böylece yakınlığa duyarlı birçok uygulama geliştirilebilir. Bu çalışmanın amacı öğrenci yoklama ya da toplantı katılım takip işlemlerinin otomatik olarak gerçekleştirilmesinde kullanılan akıllı kart, RFID, mobil uygulamalar gibi teknolojilere alternatif olarak BLE işaretçilerin kullanımını önermektir. Bu amaçla, Bluetooth düşük enerji teknolojisine sahip işaretçi cihazlar ve akıllı telefonlar kullanılarak gerçekleştirilen öğrenci yoklama sistemi sunulmaktadır. Gerçekleştirilen yoklama/katılım takip sisteminin en önemli özelliği öğrencinin katıldığı sürenin otomatik olarak izlenebilmesidir.
\end{abstract}

Anahtar Kelimeler - Otomatik yoklama sistemi, BLE işaretçi, mobil programlama, web programlama

\section{A Student Attendance System Based On Beacon and Smartphones Equipped With Bluetooth Low Energy Technology}

\begin{abstract}
Usage of BLE Beacon having Bluetooth low energy technology is rapidly increasing due to low energy consumption, low cost and support by both iOS and Android operating systems. When mobile devices such as smartphone, tablet etc. enter to deployment BLE beacon areas, they take advertising messages sent by BLE Beacon and run related mobile applications automatically. Thus, many applications sensitive to proximity can be performed. The aim of this study is to propose employing of BLE Beacons as an alternative to technologies such as smart card, RFID, mobile applications that are used to perform student polling and meeting attendance process automatically. To this end, the student attendance system using BLE Beacon and smartphones having Bluetooth low energy technology is presented. The most important attribute of the implemented attendance tracking system is monitoring the student's participation duration automatically.
\end{abstract}

Keywords-Automatic polling system, BLE beacon, mobile programming, web programming

\section{GIRISŞ (INTRODUCTION)}

Öğrencilerin derse devam takip işlemleri çoğunlukla yoklama listelerine imza atma yöntemi ile yapılmaktadır. Öğrenci sayısının çok olduğu büyük sınıflarda yoklama işlemi oldukça zaman almaktadır. Yoklamadan kaynaklanan süre kayıplarını ortadan kaldırmak veya en aza indirmek amacıyla günümüzde yoklama işlemi akıllı kart, radyo frekansı ile tanımlama (Radio Frequency IDentification, RFID) [1,2], yakın alan haberleşmesi (Near Field Communication, NFC) [3], akıllı telefon uygulamaları [4] vb. birçok farklı teknolojik altyapı kullanılarak otomatik bir şekilde gerçekleştirilmektedir. $\mathrm{Bu}$ çalışmanın ana amacı yoklama ve katılım takip işlemlerinin otomatik gerçekleştirilmesinde bu teknolojilere alternatif olarak Bluetooth düşük enerji (Bluetooth Low Energy, BLE) teknolojisine sahip işaretçi (beacon) cihazların kullanımını göstermektir.

2013 yılında Apple firması tarafindan tanıtılan ve iBeacon olarak adlandırılan işaretçi, BLE kablosuz teknolojisini kullanan kısa mesafeli, düşük güçlü, düşük maliyetli yakınlık mantığına dayalı kablosuz bildirim sağlayan elektronik cihazlardır [5]. iBeacon cihazlar konumlandırıldıkları ortamda sürekli Bluetooth paketi yayarlar ve bu paketi alan akıllı telefon gibi mobil cihazlardaki uygulamaların tetiklenerek çalışmasını sağlarlar. Her ne kadar ilk olarak Apple tarafindan 
tanıtılsa da işaretçiler hem iOS hem de Android işletim sistemine sahip mobil cihazlar ile kullanılabilmektedir. $\mathrm{Bu}$ nedenle işaretçiler BLE beacon olarak ta adlandırılmaktadır. Günümüzde birçok yabancı ve yerli firma işaretçi üretmektedir [6-9].

BLE teknolojisine sahip işaretçilerin kullanım alanlarına alış-veriş merkezlerindeki işyerlerinin ürün ve reklam tanıtımı [10], tarihi mekânların tanıtımı [11], müzeler [12], kapalı alan konum belirleme [13] gibi birçok örnek verilebilir.

$\mathrm{Bu}$ çalışmada güncel bir teknoloji olan BLE işaretçi ve akıllı telefonlardan yararlanarak geliştirilen bir öğrenci yoklama sistemi sunulmaktadır. Geliştirilen sistem devam takip işlemlerinin otomatik olarak gerçekleştirilmesi, öğrencinin katılım süresinin saat bazında tam olarak belirlenmesi, yoklama işlemi için harcanan sürenin kısaltılması gibi birçok avantaj sunmaktadır. Bununla birlikte kullanıcıların BLE teknolojisini destekleyen mobil cihazlara sahip olma zorunluluğu sistemin bir dezavantajı olarak göze çarpmaktadır. Ancak BLE teknolojisinin hızla yaygınlaşması ve yeni nesil telefonların bu teknolojiyi standart olarak sunması dikkate alındığında bu dezavantajın yakın bir zamanda ortadan kalkacağı aşikârdır.

Makalenin organizasyonu şu şekildedir. Bölüm 2'de BLE teknolojisi ve BLE işaretçi tanıtılmaktadır. Bölüm 3'de gerçekleştirilen öğrenci yoklama sisteminin mimarisi, çalışma prensibi, yazılım alt yapısı ve kullanımı sunulmaktadır. Son bölümde ise sonuçlar ve değerlendirmeler verilmektedir.

\section{BLUETOOTH DÜŞÜK TEKNOLOJISIINE SAHİP IŞARETÇI EQUIPMENT WITH BLUETOOH LOW TECHNOLOGY) \\ ENERJi \\ (BEACON ENERGY}

İşaretçi (Beacon) cihazlar, Bluetooth 4.0 ya da Bluetooth smart olarak ta bilinen BLE teknolojisi ile donatılmış, düşük güçlü ve düşük maliyetli elektronik vericilerdir. Şekil 1'de phoneist firmasının bluegiga BLE113 çipine sahip işaretçi cihazı görülmektedir. bluegiga BLE 113 çipi gönderim işleminde maksimum 18,2 mA, alımda maksimum $14.3 \mathrm{~mA}$, uyku modunda ise $0,4 \mu \mathrm{A}$ enerji tüketimine sahiptir [14].

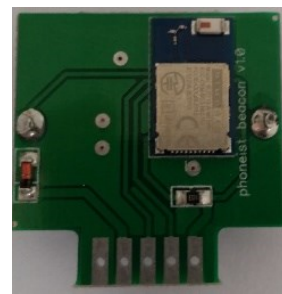

Şekil 1. İşaretçi cihaz (The beacon device)
BLE, Bluetooth teknolojisi gibi $2.4 \mathrm{GHz}$ frekans bandında, Frekans Atlamalı Geniş Spektrum (Frequency Hopping Spread Spectrum) tekniğini kullanarak $1 \mathrm{Mbit} / \mathrm{s}$ veri iletim hızında haberleşme sağlar. Ancak güç tüketimi diğer Bluetooth sürümlerine göre oldukça düşüktür. BLE teknolojisi kolay kullanım açısından internet bağlantısı, sağlık, spor, genel sensör türleri iletişimi gibi 7 profil desteği sunar [15]. Tablo 1'de kısa mesafeli kablosuz haberleşme teknolojilerinin karşılaştırılması verilmektedir. BLE teknolojisi diğer kablosuz teknolojiler ile karşılaştırıldığında çok düşük güç tüketimine sahiptir $[15,17,18]$.

Tablo 1. Kısa mesafeli kablosuz haberleşme teknolojilerinin karşılaştırılması

(A comparison of short-range wireless communication technologies)

\begin{tabular}{|c|c|c|c|c|}
\hline $\begin{array}{l}\text { Kablosuz } \\
\text { teknoloji }\end{array}$ & $\begin{array}{c}\text { Veri } \\
\text { iletim } \\
\text { hizl }\end{array}$ & $\begin{array}{l}\text { Çalışma } \\
\text { frekansl }\end{array}$ & $\begin{array}{c}\text { Kapsama } \\
\text { alanı }\end{array}$ & $\begin{array}{r}\text { Ortalama } \\
\text { ă ömrü }\end{array}$ \\
\hline $\begin{array}{c}\text { BLE } \\
\text { IEEE802.15.1 } \\
(\mathrm{v} 4)\end{array}$ & $1 \mathrm{Mbit} / \mathrm{s}$ & $2.4 \mathrm{GHz}$ & $10-50 \mathrm{~m}$ & $1-2$ y1l \\
\hline $\begin{array}{c}\text { Bluetooth } \\
\text { IEEE802.15.1 }\end{array}$ & $\begin{array}{c}1-24 \\
\mathrm{Mbit} / \mathrm{s}\end{array}$ & $2.4 \mathrm{GHz}$ & $10-100 \mathrm{~m}$ & Gün - ay \\
\hline $\begin{array}{c}\text { UWB } \\
\text { IEEE802.15.3a }\end{array}$ & $\begin{array}{c}110-480 \\
\mathrm{Mbit} / \mathrm{s}\end{array}$ & $\begin{array}{c}3.1-10.6 \\
\mathrm{GHz}\end{array}$ & $10 \mathrm{~m}$ & $1-2$ y1l \\
\hline $\begin{array}{c}\text { ZigBee } \\
\text { IEEE802.15.4 }\end{array}$ & $\begin{array}{c}20-250 \\
\text { Kbit/s }\end{array}$ & $2.4 \mathrm{GHz}$ & $100 \mathrm{~m}$ & $\begin{array}{c}6 \text { ay }- \\
1 \text { y1l } \\
\end{array}$ \\
\hline Z-Wave & $\begin{array}{c}40 \\
\text { Kbit/s }\end{array}$ & $\begin{array}{c}868 / 908 \mathrm{MHz} \\
2.4 \mathrm{GHz}\end{array}$ & $100 \mathrm{~m}$ & $1 \mathrm{y} 1 \mathrm{l}$ \\
\hline
\end{tabular}

İşaretçi cihazlar, Bluetooth paket (BLE Advertising) olarakta adlandırılan belirli sıklıkta kablosuz sinyaller yayarlar. Bu sinyaller BLE destekleyen Android ve iOS işletim sistemine sahip mobil cihazlar tarafından alınır. Mobil cihazlardaki uygulamalar, işaretçi cihazlardan alınan kablosuz sinyaller ile tetiklenir/çalıştırılır. Böylece mobil uygulamalar yalnızca işaretçi cihazın kapsama alanına girildiğinde çalışmaktadır. $\mathrm{Bu}$ durum yani etkileşim kullanıcılara büyük kolaylıklar sunmaktadır [16].

İşaretçi cihazlardan yayılan Bluetooth sinyaller UUID (Universal Unique Identifier), üst adres (major), alt adres (minor) ve sinyal gücü bilgilerini içermektedir. UUID, 16 bayt uzunluğundadır. Tüm ağı tanımlamak için kullanılır ve genellikle üretici bilgisini içerir. Major ve Minor adresler ise 2 bayt uzunluğundadır. Örneğin bir binaya işaretçi yerleștirirken UUID olarak üretici bilgisi ya da bina bilgisi, major olarak kat bilgisi, minor olarak ise oda numarası verilebilir. Böylelikle işaretçi yerleştirilen konumlar kolayca tanımlanabilir ve takip edilebilir [16].

iOS işletim sistemi ile çalışan işaretçi cihazlar ibeacon olarak adlandırılmaktadır [5]. Google Beacon Eddystone [19] ismini verdiği teknoloji ile BLE işaretçi alanında mobil uygulama etkileşiminin yanısıra URL çalıştırma özelliğini de getirerek işaretçi alanında önemli bir yenilik sunmaktadır. 


\section{BLE IŞARETÇİ VE AKILLI TELEFON TEMELLİ ÖĞRENCI YOKLAMA SISTEMİ (A STUDENT ATTENDANCE SYSTEM USING BLE BEACON AND SMARTPHONE)}

\subsection{Sistem Mimarisi (System Arhitecture)}

Şekil 2'de öğrenci yoklama sisteminin gerçekleştirilmesinde kullanılan sistem mimarisi ve sistem bileşenleri görülmektedir. Şekilden görüldüğü üzere öğrenci yoklama sistemi; (i) işaretçi, (ii) işaretçinin yaydığı BLE sinyalleri ile etkileşimde bulunan mobil uygulama ve akıllı telefon, (iii) veritabanı ve (iv) web temelli devam takip yazılımı ile bunların haberleşmesini sağlayan (v) web servisleri içeren sunucu olmak üzere 5 temel bileşenden oluşmaktadır.

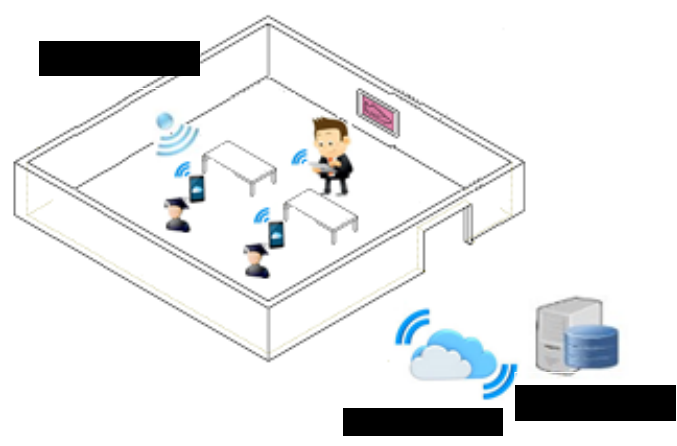

Şekil 2. Gerçekleştirilen sistem mimarisi (The implemented system arhitecture)

Yoklama sisteminin genel çalışması şu şekildedir. İlk olarak kullanıcılar, mobil uygulamaya kullanıcı adı ve şifre ile giriş yapmalıdırlar. Sınıflara yerleştirilen işaretçiler, kullanıcıların akıllı telefonlarındaki mobil uygulamaları ile etkileşimde bulunmakta, ders programları ve kullanıcının sınıfta bulunma sürelerini dikkate alarak yoklama işlemini otomatik bir şekilde gerçekleştirmektedir. Mobil yoklama uygulaması $\mathrm{WiFi} / 3 \mathrm{G}$ gibi kablosuz teknolojiler aracılığıly sunucudaki yoklama işlemini yerine getirmektedir. Ayrıca kullanıcılar akademik bilgi sisteminin web arayüzünü kullanarak derse katılım durumlarını takip edebilmektedir. Geliştirilen sistemin çalışması Şekil 3'de verilen dizge diyagramında özetlenmektedir.

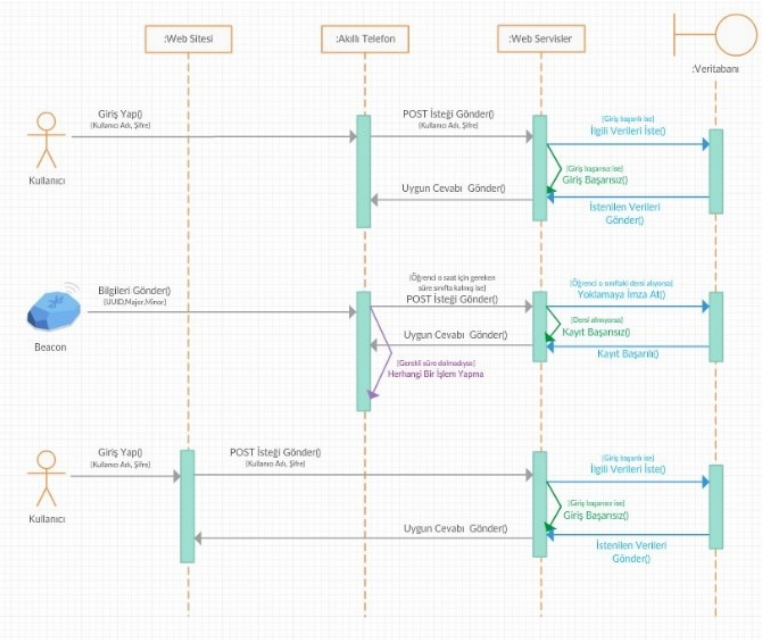

Şekil 3. Gerçekleştirilen sistemin dizge diyagramı (The sequence diagram of the implemented system)

Şekil 4'te geliştirilen yoklama sisteminin veritabanı modeli sunulmaktadır. Veritabanı olarak MySQL veritabanı kullanılmıştır. Şekilden de görüldüğü üzere veritabanı birbiriyle ilişkili öğrenciler, öğretmenler, dersler, programlar ve yoklama vb. bilgilerini içeren toplam 8 tablodan oluşmaktadır.

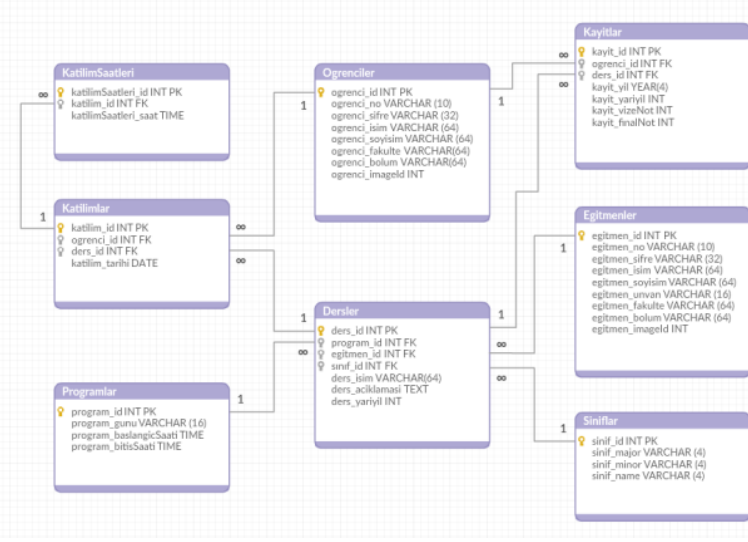

Şekil 4. Veritabanı modeli (Database model)

3.2. Android Tabanlı Mobil Yoklama Uygulamas1 (Android-Based Mobile Polling Application)

Android tabanlı mobil uygulama Android Studio editöründe gerçekleştirilmiştir. Geliştirilen mobil yoklama uygulamasının amacı, kullanıcı işaretçi cihazların kapsama alanına girdiğinde, kullanıcının bulunduğu konumu anlamakta ve bu konumla ders programını ilişskilendirerek yoklama işleminin otomatik olarak gerçekleştirilmesini sağlamaktır.

Şekil 5'de gerçekleştirilen mobil yoklama uygulamasının kullanıcı arayüzleri görülmektedir. Kullanıcı adı ve şifre ile mobil uygulamaya giriş yapıldıktan sonra ilk olarak kullanıcı bilgileri gelmektedir. Mobil uygulama arka 
planda çalışmakta ve BLE teknolojisini kullanarak işaretçi tarama işlemine devam etmektedir. İşaretçinin bulunduğu sınıfa gelindiğinde ise uygulama kullanıcının sınıfa geliş ve sınıfta bulunma sürelerini, ders programı ile kontrol ederek yoklamayı saat saat gerçekleştirmektedir. Uygulama, sinıfta bulunulan süre bir ders saati süresinin en az yarısı olduğunda o saat için yoklama işlemini gerçekleştirmektedir. Bu durum kullanıcıya şekilde de görüldüğü üzere bildirim mesajları ile aktarılmaktadır. Ayrıca bu bildirim mesajı ders bilgilerini içeren ayrıntılı bir şekilde de mesaja tıklanarak görülebilmektedir.
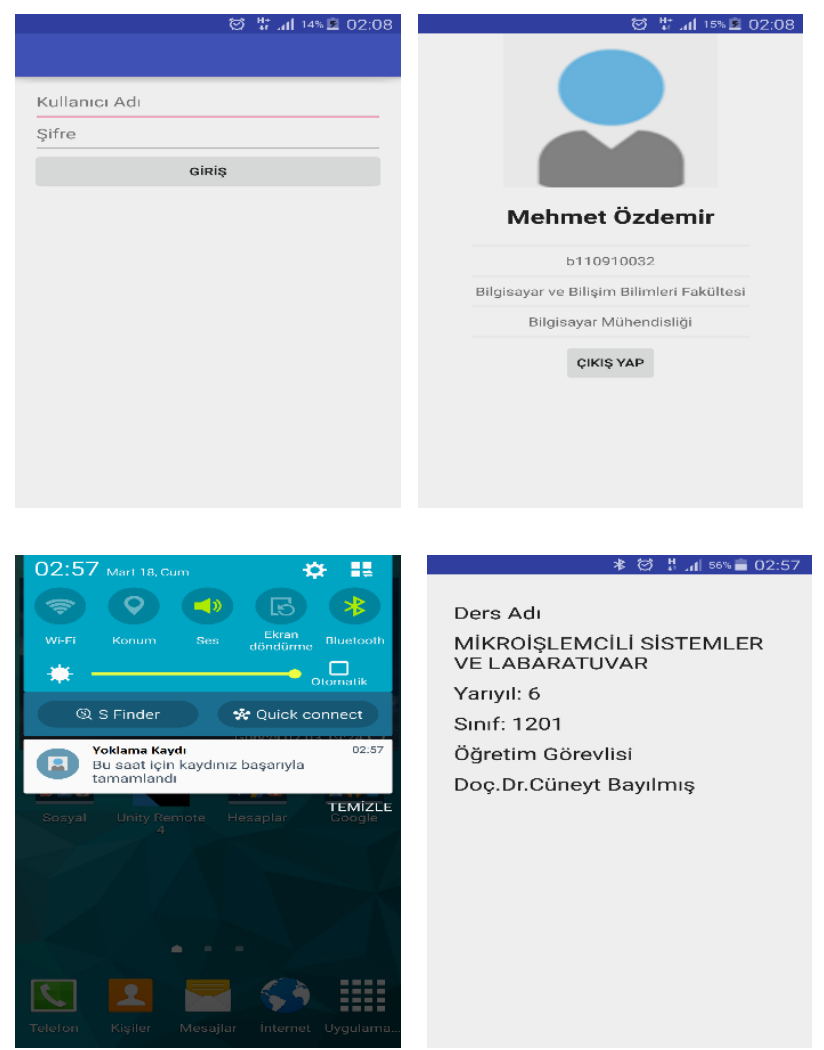

Şekil 5. Mobil yoklama uygulama arayüzleri (Interfaces of mobile polling application )

\subsection{Web Temelli Devam Takip Yazılımı (Web-based Attendance Tracking Software)}

Web temelli devam takip yazılımı, kullanıcının derse katılım durumlarının takibini/izlenmesini sağlamayı amaçlamaktadır. $\mathrm{Bu}$ yazılımının tasarım ve geliştirilmesinde bootstrap, php, html teknolojileri kullanılmıştır.

Hem öğretmen hem de öğrenci web temelli devam takip yazılımına Şekil 6'da görülen aynı arayüz üzerinden kullanıcı adı ve şifre bilgileri ile girmektedir.

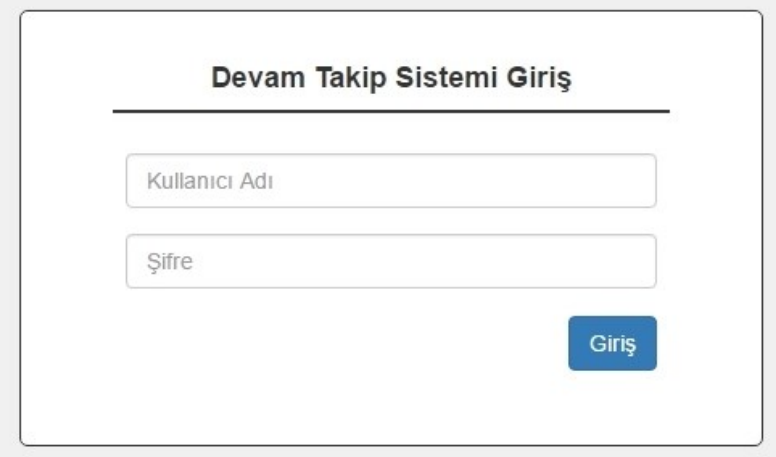

Şekil 6. Web temelli devam takip yazılımı giriş ekranı (Login page of web-based attendance tracking software)

Öğretmen web temelli devam takip sistemine giriş yaptığında vermekte olduğu derslerini ve derslerine ait gün, saat, derslik bilgilerini görmektedir (Şekil 7). Bu arayüz aracılığıyla verilmekte olan herhangi bir ders seçildiğinde Şekil 8'deki arayüz gelmektedir. $\mathrm{Bu}$ arayüzde ilgili dersi alan öğrenciler ve öğrencilerin toplam katılım yüzde oranları görülmektedir. Yoklama listesindeki herhangi bir öğrenci tıklandığında ise Şekil 9'da görüldüğü gibi haftalık ayrıntılı katılım bilgileri görülmektedir. Örneğin Mehmet Özdemir isimli öğrenci 23 Şubat tarihli dersin ilk iki saatinde derse katılmış, 3. saat derste bulunmamış, 4. saat tekrar derse katılmıştır. Sonuç olarak ilgili hafta için ders katılım yüzdesi $\% 75$ 'dir.

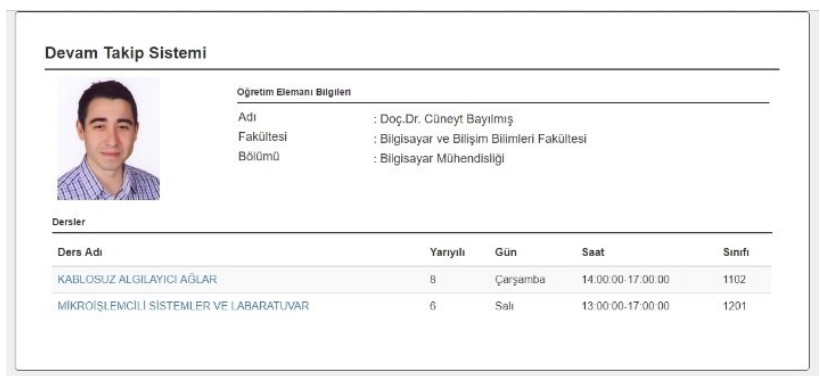

Şekil 7. Web temelli devam takip yazılımında öğretmen arayüzü

(Teacher interface in web-based attendance tracking software)

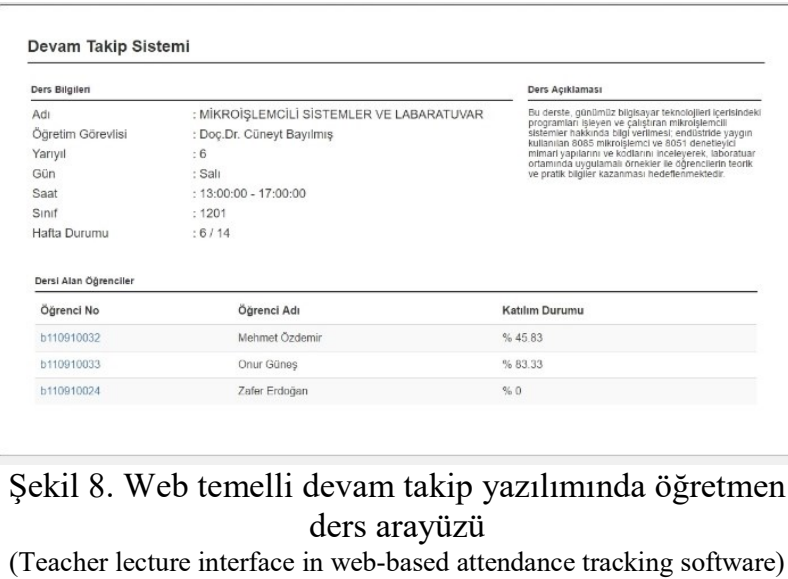




\section{SONUÇLAR (CONCLUSIONS)}

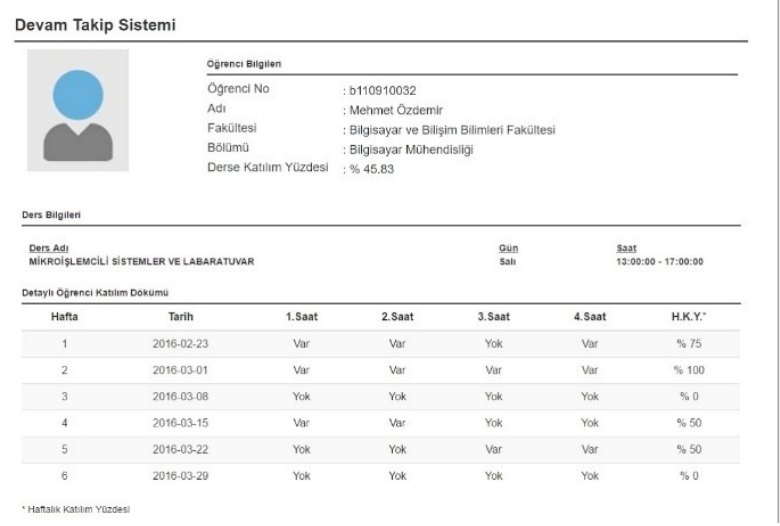

Şekil 9. Web temelli devam takip yazılımında ders arayüzünün detaylı gösterimi

(Teacher lecture interface detail in web-based attendance tracking software)

Öğrenci web temelli devam takip sistemine giriş yaptığında ise almakta olduğu dersleri, gün, saat ve derslik bilgilerini görmektedir (Şekil 10). Derslerden biri tıklandığında o derse ait ayrıntılı katılım bilgileri ve yüzdeleri görülmektedir (Şekil 11).

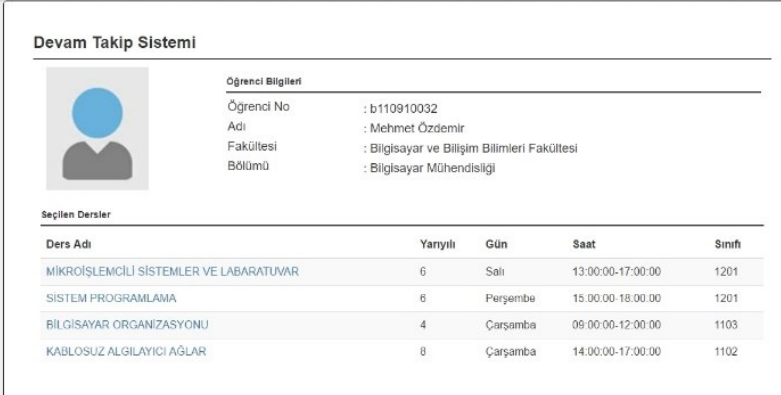

Şekil 10. Web temelli devam takip yazılımında öğrenci arayüzü

(Student interface in web-based attendance tracking software)

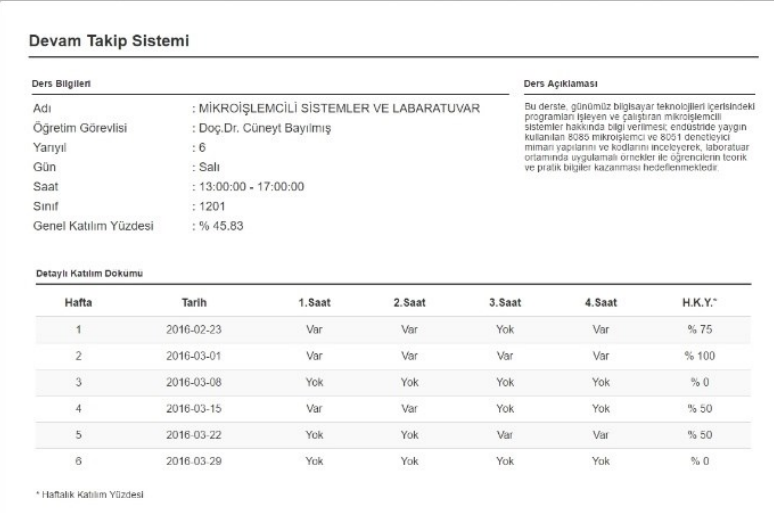

Şekil 11. Web temelli devam takip yazılımında öğrencinin ilgili derse ait arayüzü

(Student lecture interface in web-based attendance tracking software)
iOS ve Android işletim sistemli mobil kullanıcılar, BLE kablosuz haberleşme teknolojisini kullanan işaretçilerin kapsama (etkileşim) alanına girdiğinde uygulama çalıştırma, içerik sunma gibi hizmetler almaktadır. İşaretçiler reklam, lojistik, ürün, tarihi eser ve yerlerin tanıtımı, navigasyon gibi çok geniş uygulama alanı sunmaktadır.

Bu çalışmada, kullanımı hızla yaygınlaşan ve güncel bir teknoloji olan işaretçilere dayalı olarak geliştirilen yoklama ve katılım sistemi sunulmuştur. Geliştirilen sistem kullanıcıların işaretçiler ile etkileşimde bulunduğu konum ve süreye göre katılımlarını takip etmeyi sağlamaktadır. Böylelikle öğrencinin hangi saat sınıfta olduğu hangi saat ayrıldığı izlenebilmektedir.

Geliştirilen öğrenci yoklama sistemi yapılacak küçük değişiklikler ile kurumsal firmaların toplantı takip ve değerlendirme süreçlerinde de kullanılabilir. Firma sahibi ya da proje yöneticileri, toplantılara katılanları, katılma sürelerini raporlama imkanına sahip olacaktır.

Geliştirilen sistemin en büyük dezavantajı ise kullanıcıların BLE teknolojisine sahip mobil cihaz kullanımıdır. Günümüzde yeni cihazların çoğunda BLE teknolojisinin standart olarak sunulması bu dezavantajin hızla giderileceğini göstermektedir.

\section{KAYNAKLAR (REFERENCES)}

[1] M. Kapıdere, S. Avcu, "Akıllı Kart İle Öğrenci Yoklama Sistemi ve Uygulaması", Akademik Bilişim, Mersin, Türkiye, 2014.

[2] F. Bektaş, A. Sondaş, "RFID Modülü ile Kapı GirişÇıkış Takip Sistemi”, Ulusal Mühendislik Araştırmaları Sempozyumu, Düzce, Türkiye, 2015.

[3] M. A. Ayu, B. I. Ahmad, "TouchIn: An NFC Supported Attendance System in a University Environment", International Journal of Information and Education Technology, Vol. 4, No. 5, 448-453, 2014.

[4] Internet: Sabis: Sakarya Üniversitesi Bilgi Sistemi, http://www.sabis.sakarya.edu.tr, 16.3.2016.

[5] Internet: Apple: iBeacon for developers, https://developer.apple.com/ibeacon/, 16.3.2016.

[6] Internet: Estimote beacon, https://estimote.com, 16.3.2016.

[7] Internet: Blesh beacon, https://www.blesh.com, 16.3.2016. 
[8] Internet: Boni beacon, https://www.boni.me, 16.3.2016

[9] Internet: Phoneist beacon, https://www.phoneist.com, 16.3.2016.

[10] P. Burzacca, M. Mircoli, S. Mitolo, A. Polzonetti, "iBeacon technology that will make possible Internet of Things", International Conference on Software Intelligence Technologies and Applications \& International Conference on Frontiers of Internet of Things 2014, Hsinchu, Taiwan, 159-165, 4-6 December 2014.

[11] A. Ito, Y. Hiramatsu, H. Hatano, M. Sato, M. Fujii, Y. Watanabe, F. Sato, A. Sasaki, "Navigation System for Sightseeing using BLE Beacons in a Historic Area", IEEE 14th International Symposium on Applied Machine Intelligence and Informatics, Herl'any, Slovakya, 171-176, 21-23 January 2016.

[12] Z. He, B. Cui, W. Zhou, S. Yokoi, “A proposal of interaction system between visitor and collection in museum hall by iBeacon", The 10th International Conference on Computer Science \& Education (ICCSE 2015), Cambridge University, UK, 427-430, 2224 July 2015.

[13] X. Lin, T. Ho, C. Fang, Z. Yen, B. Yang, F. Lai, “A Mobile Indoor Positioning System Based on iBeacon Technology", 37th Annual International Conference of the IEEE Engineering in Medicine and Biology Society (EMBC), Milan, Italy, 4970-4973, 25-29 August 2015.
[14] Internet: Bluegiga BLE113, https://www.bluegiga.com/en-US/products/ble113bluetooth-smart-module/, 17.3.2016.

[15] M. Radhakrishnan, A. Misra, R. K. Balan, Y. Lee, "Smartphones \& BLE Services: Empirical Insights", 12th International Conference on Mobile Ad Hoc and Sensor Systems, Dallas, USA, 226-234, 19-22 October 2015.

[16] M. Köhne, J. Sieck, "Location-based Services with iBeacon Technology", Second International Conference on Artificial Intelligence, Modelling and Simulation, Madrid, Spain, 315-321, 18-20 November 2014.

[17] D. N. Malayeri, "Review of UWB Technology Specifiactions and Benefits As a Powerful Technology to Develop Power Distribution Grid Automation Techniques", 16th Conference on Electrical Power Distribution Networks, İran, 1-7, 19-20 April 2011.

[18] S. Gajjar, N. Choksi, M. Sarkar, K. Dasgupta, "Comparative analysis of Wireless Sensor Network Motes", International Conference on Signal Processing and Integrated Networks (SPIN), Delhi, India, 426-431, 20-21 February 2014.

[19] Internet: Google beacon eddystone, https://developers.google.com/beacons/, 16.3.2016. 